\title{
ARTÍCULOS
}

Sometido 04.05.2015. Aprobado 01.09.2015

Evaluado por el sistema double blind review. Editor Científico: Antonio Navarro-García

DOI: http://dx.doi.org/10.1590/So034-759020160202

\section{LAS PREFERENCIAS DEL CONSUMIDOR USANDO EL MÉTODO DE MÁXIMAS DIFERENCIAS}

\author{
As preferências do consumidor aplicando o método de máximas diferenças \\ Consumer preferences using the maximum difference method
}

\begin{abstract}
RESUMEN
Este estudio tiene como objetivo identificar los atributos más importantes que el consumidor limeño utiliza en el proceso de elección del vino. Adicionalmente, se busca identificar diferencias entre segmentos en estas preferencias. La metodología consiste en una encuesta a consumidores de vino usando el método de máximas diferencias. El estudio es único en América Latina, donde existe una brecha importante en el mercado del vino y donde el método de máximas diferencias es inexplorado por administradores e investigadores. Los resultados muestran que los atributos más importantes se relacionan con la experiencia previa del consumidor con el vino, el maridaje y las recomendaciones. Este artículo presenta las implicancias para los administradores sobre sus estrategias de marketing a nivel de Lima, generaciones etarias, estratos socioeconómicos y género.
\end{abstract}

PALABRAS CLAVE | Consumidor de vino, método de máximas diferencias, preferencias, importancia de los atributos, desarrollo de nuevos produtos.

\section{RESUMO}

O estudo tem como objetivo identificar os atributos mais importantes utilizados pelo consumidor limenho no processo de escolha de vinhos. Além disso, busca-se identificar diferenças entre segmentos nestas preferências. A metodologia consiste em um levantamento dos consumidores de vinho por meio do método de diferenças máximas. O estudo é único na América Latina, onde existe uma importante lacuna no mercado de vinho e onde o método de máximas diferenças é inexplorado pelos empresários e acadêmicos. Os resultados mostram que os atributos mais importantes estão relacionados à experiência prévia consumidor com vinhos e às recomendações. $O$ artigo apresenta as implicações aos empresários para suas estratégias de marketing no âmbito de Lima, gerações etárias, estratos socioeconômicos e gênero.

PALAVRAS-CHAVE I Consumidor de vinho, método de máximas diferenças, preferências, importância dos atributos, desenvolvimento de novos produtos.

\section{PABLO FARÍAS}

pfarias@fen.uchile.cl

Profesor de la Universidad de Chile, Facultad de Economía y Negocios Santiago, Chile

\section{BRUNO FISTROVIC}

bfistrovic@fen.uchile.cl

Magister en Marketing por la

Universidad de Chile, Facultad de

Economía y Negocios - Santiago, Chile

\section{ABSTRACT}

This study aims to identify the most important attributes employed by consumers in Lima in the process of choosing wine. Furthermore, it seeks to identify differences between segments in these preferences. The methodology consists of a survey of wine consumers using the maximum difference method. The study is unique in Latin America, where there is a major gap in the wine market and where the maximum difference method is unexplored by managers and researchers. The results show that the most important attributes are related to the consumer's prior experience with the wine, pairings, and recommendations. This article presents the implications for managers and marketing strategies in the context of Lima by age, socioeconomic, and gender group.

KEYWORDS I Wine consumer, maximum difference method, preferences, importance of attributes, development of new products. 


\section{INTRODUCCIÓN}

A diferencia de otras industrias, la producción del vino está profundamente relacionada a la tradición y a la agricultura, como punto central de la cadena de valor. Esto podría explicar la dificultad aparente en adoptar un enfoque de orientación al mercado (Bruwer, Li, \& Reid, 2002). ¿Imagina usted que uno de los vinos más exitosos de la bodega Chateau Diana, lleva en su etiqueta frontal la figura de un zombi al estilo "The Walking Dead"? Las ventas del llamado "Zombie Zin" han crecido exponencialmente gracias a un enfoque en los jóvenes de California (Paulas, 2014). El especialista en marketing debe encontrar la manera de entender las necesidades, deseos y demandas de los consumidores (Casini, Corsi, \& Goodman, 2009). Es por ello que conocer las preferencias de los distintos segmentos es relevante para definir estrategias de marketing más efectivas para accionar en el mercado (Goodman, Lockshin, \& Cohen, 2005).

Perú es uno de los mercados con mayor potencial de crecimiento en América Latina (Guzmán, 2014). Esto es gracias al crecimiento económico, el desarrollo de la gastronomía y el turismo, y a la expansión de los canales de comercialización relacionados al vino (SNI, 2014). La capital de Perú, Lima, cuenta con más de 8,5 millones de habitantes. Lima además es la ciudad con mayor consumo per cápita de vino de Perú, transformando a Lima en un mercado muy atractivo tanto por su tamaño como por su consumo per cápita (Gestión, 2013). Por lo tanto, el presente trabajo busca examinar las preferencias de los consumidores limeños de vino sobre un conjunto de atributos que influyen en el proceso de elección del vino. Además, este trabajo busca identificar diferencias entre distintos segmentos de consumidores con la finalidad de definir estrategias de marketing más efectivas y enfocadas. Para ello, se plantea utilizar el método de máximas diferencias (MaxDif). El estudio es único en América Latina, donde existe una brecha importante en el mercado del vino y donde el método de máximas diferencias es inexplorado por administradores e investigadores.

La tarea es complicada en la industria del vino por dos razones fundamentales. En primer lugar, los consumidores están al frente de una gran cantidad de información, más de cien mil marcas en el mundo, un sinfín de uvas, países y regiones de origen (Goodman et al., 2005). El vino presenta un conjunto extenso de señales, lo cual puede generar confusión en el proceso de elección (Bernabéu, Díaz, Olivas, \& Olmeda, 2012). Por ejemplo, señales extrínsecas como la marca, el precio, la etiqueta, el lugar de origen, y señales intrínsecas como el sabor, los aromas y el contenido de alcohol, son difíciles de evaluar sin degustar el vino (Cohen, 2009). En segundo lugar, el nivel de fragmentación en la industria no le permite a las empresas dirigirse a todos los segmentos de la población. Es mejor que cada empresa, de acuerdo a su disposición, se concentre en segmentos específicos del mercado con el objetivo de desarrollar una gestión de marketing eficiente. Para ello, comprender los atributos que influyen en la elección del vino de distintos segmentos es un paso fundamental para la estrategia (Casini et al., 2009).

Este estudio aplica un método reciente (MaxDif) para evaluar las preferencias del consumidor de vino, y que ha demostrado tener éxito (Cohen \& Neira, 2003; Flynn, Louviere, Peters, \& Coast, 2007; Goodman et al., 2005) al superar un conjunto de limitaciones en la medición (Finn \& Louviere, 1992). Este método en la actualidad es muy utilizado por los investigadores y administradores en países desarrollados (Louviere, Lings, Islam, Gudergan, y Flynn, 2013), sin embargo, en América Latina ha sido poco o nada explorado. Además, este estudio efectuado en la ciudad de Lima es único en América Latina, donde existe una brecha importante en el conocimiento del consumidor de vino.

Las secciones siguientes presentan la revisión de la literatura, la metodología de la investigación y los resultados. Finalmente, la última sección presenta las conclusiones, implicancias y limitaciones de la investigación.

\section{REVISIÓN DE LA LITERATURA}

\section{El consumo de vino en Perú}

Las cifras de consumo de vino en América Latina crecen en casi todos los mercados. Países como Colombia, Chile, Guatemala, México o Perú muestran importantes ascensos en los volúmenes de consumo. El consumo per cápita de vino en el Perú se ha duplicado de 0,96 litros en el año 2006 a 2 litros en la actualidad (SNI, 2014). El mercado peruano tiene un importante potencial de crecimiento (Guzmán, 2014), ya que en países como Uruguay, Argentina y Chile, el consumo per cápita es de 29, 24 y 17 litros anuales, respectivamente, $y$ en varios países europeos el consumo per cápita supera los 30 litros anuales. Los consumidores peruanos están ávidos de recibir vinos distintos, probar nuevas variedades y, sobre todo, se han dado cuenta que en el Perú se puede producir vino de calidad y comercializarlo a otros mercados (El Comercio, 2014). La demanda de vinos en Perú ha crecido gracias a una mejora en los ingresos de la población y al desarrollo de una oferta gastronómica y turística importante. Además, la expansión de los canales de distribución no solo ha permitido el surgimiento de marcas peruanas sino también el ingreso de una amplia variedad de marcas importadas a diversos precios, gracias a las bajas barreras de comercialización a productos del exterior (SNI, 2014). 
Se estima que el consumo de vinos en el Perú en 2014 fue de 63 millones de litros y que el $80,3 \%$ correspondió a vinos nacionales y $19,7 \%$ a vinos importados. En los últimos diez años la importación de vinos ha crecido a una tasa anual de $12,8 \%$, habiendo triplicado su valor en términos FOB. Sin embargo, el volumen importado en litros no ha crecido en la misma magnitud (1,5 veces). Esto es porque están entrando vinos de mayor valor y calidad al mercado. Entre los años 2004 y 2013 , los precios promedio crecieron un $89,1 \%$. Efectivamente, el valor unitario promedio de los vinos importados era de US\$ 2,02 en 2004, y se incrementó a US\$3,82 por litro en 2013. Perú es uno de los principales mercados para los vinos argentinos en Latinoamérica, con 366 marcas argentinas y 46\% de participación en valor en vinos importados. Asimismo, los vinos chilenos en Perú alcanzaron una participación en valor del $31 \%$ en vinos importados. Un tercer actor importante en el mercado peruano son los vinos españoles los que alcanzaron una participación en valor del $11 \%$ de los vinos importados. Respecto al comportamiento mensual de las importaciones de vino en términos de volumen, se puede observar una marcada estacionalidad. En los primeros meses del año, se importan menores cantidades de vino; mientras que, en los últimos meses, la importación se incrementa en forma significativa, preparándose para la campaña navideña (SNI, 2014).

Lima con más de 8,5 millones de habitantes es la ciudad con mayor consumo per cápita de vino de Perú, transformando a Lima en un mercado muy atractivo tanto por su tamaño como por su consumo per cápita (Gestión, 2013). Sin embargo, las provincias del interior del país también se posicionan como consumidores de vino, en particular las ciudades de Chiclayo, Trujillo, Arequipa, Huancayo e Ica (El Comercio, 2014).

\section{Importancia de los atributos}

La importancia de los atributos para la elección de vino pueden variar de acuerdo a las características de los consumidores (Bernabéu et al., 2012). Como consecuencia, en esta sección se revisa la literatura existente y se plantean hipótesis tanto a nivel de la ciudad de Lima como a nivel de segmentos de consumidores por edad, estrato socioeconómico y género.

\section{Importancia de los atributos para los consumidores limeños}

\section{La importancia del haber degustado el vino previamente y las recomendaciones}

Balestrini y Gamble (2006) demostraron que las recomendaciones de un amigo, personal de la tienda o del sommelier contribuyen a reducir el riesgo percibido por el consumidor. En la misma línea, Manske y Cordua (2005) determinaron que el papel del sommelier es muy importante, debido a que puede comunicar las características del vino eficientemente, ayudando a los consumidores a tomar una mejor decisión de compra. Goodman (2009) investigó los atributos más importantes para los consumidores de vino en doce países. Los atributos como el haber degustado el vino previamente y las recomendaciones fueron muy importantes en la mayoría de los mercados, con excepción de algunos países, donde la marca (China y Brasil) o el maridaje (Francia e Italia) fueron los atributos más importantes para los consumidores de vino. No obstante, Casini et al. (2009) observan que haber degustado el vino previamente es uno de los atributos más importantes para los consumidores italianos. Por otro lado, De-Magistris, Gracia, y Albisu (2014) señalan que la denominación de origen, el año de la vendimia, el maridaje y el haber leído del vino anteriormente, se consideran atributos más importantes en los países desarrollados. Por otro lado, De-Magistris et al. (2014) señalan que la variedad de uva, el país de origen, las recomendaciones y el haber degustado el vino previamente son los atributos más importantes en los países emergentes. Siguiendo a la evidencia empírica internacional y especialmente a la observada en países emergentes, para los consumidores limeños dos atributos muy importantes al momento de elegir vino deben ser el haber degustado el vino previamente y las recomendaciones. Por lo tanto, se plantean las dos primeras hipótesis:

H1: Cuando compran un vino, para los consumidores limeños el haber degustado el vino previamente está entre los atributos más importantes.

H2: Cuando compran un vino, para los consumidores limeños la recomendación está entre los atributos más importantes.

\section{La importancia de la variedad de uva y el país de origen}

Varios estudios muestran que la variedad de uva es otro atributo importante al momento de elegir un vino (Lockshin y Hall, 2003; Felzensztein, Hibbert, \& Vong, 2004; Goodman et al., 2005; Thomas y Pickering, 2003). Por otro lado, la zona (región, país) de origen es uno de los atributos más importantes en la compra de vino por parte de los consumidores australianos (Batt y Dean, 2000) y chinos (Balestrini y Gamble, 2006). Asimismo, De-Magistris et al. (2014) señala que la variedad de uva y el país de origen son dos atributos muy importantes en los países emergentes. En Perú, la mayoría de los consumidores prefiere variedades dulces o semisecas. Conforme crece la cultura enológica, los tipos secos han empezado a tener más demanda (Perú21, 2011). Esto sugiere que para los consumidores limeños la variedad de uva podría ser un atributo muy importante. Por otro 
lado, el país de origen puede ser un atributo muy importante para los consumidores en América Latina si consideramos el éxito que han logrado en varias categorías de producto las marcas globales en la región (Farías, 2015). Por lo tanto, para los consumidores limeños dos atributos muy importantes al momento de comprar vino deben ser la variedad de uva y el país de origen. Por lo tanto, se plantean las siguientes dos hipótesis:

H3: Cuando compran un vino, para los consumidores limeños la variedad de uva está entre los atributos más importantes.

$\mathrm{H}_{4}$ : Cuando compran un vino, para los consumidores limeños el país de origen está entre los atributos más importantes.

\section{La importancia de la oferta/promoción/descuento de la tienda o restaurante}

Una estrategia de entrada que han utilizado varias marcas de vino en el mundo incluido Perú son las ofertas/promociones/ descuentos en la tienda y restaurantes. Atkin, Nowak, y Garcia (2007) indican que las promociones en el punto de venta son comúnmente consideradas por los consumidores de vino, y que los estimula a comprar. En América Latina, las promociones en la tienda han demostrado influir sobre las preferencias de los consumidores (Manzur, Olavarrieta, Hidalgo, \& Farías, 2013; Manzur, Olavarrieta, Hidalgo, Farías, \& Uribe, 2011; Olavarrieta, Hidalgo, Manzur, \& Farías, 2012). A esto se suma el estudio de Arellano Marketing (2014) en Perú, quien muestra que el 38\% de los consumidores prefieren los descuentos al momento de hacer compras, lo cual podría ser determinante en la elección de vino. En orden a investigar la importancia de la oferta/promoción/descuento de la tienda o restaurante para los consumidores limeños al momento de comprar un vino, se plantea una quinta hipótesis:

$\mathrm{H}_{5}$ : Cuando compran un vino, para los consumidores limeños la oferta/promoción/descuento es uno de los atributos más importantes.

\section{Importancia de los atributos a nivel de las generaciones etarias}

La generación $\mathrm{Y}$, también conocida como la generación del milenio o millennials, es la cohorte demográfica que sigue a la generación X. Sus edades en la actualidad están entre los 18 y 34 años. Bernabéu et al. (2012) observa que la producción orgánica es un atributo relativamente importante para los consumidores jóvenes. Adicionalmente, los jóvenes han demostrado dar una mayor importancia al precio del vino y al diseño (Thach \& Olsen, 2006). En España, García, Barrena, y Grande (2013) observan que los jóvenes están más preocupados por un estilo saludable, y que solo un pequeño grupo de ellos tiene una vaga idea de las denominaciones de origen. En forma similar, el tiempo de envejecimiento no parece condicionar mucho en la elección del vino en los jóvenes (Bernabéu et al., 2012). Como consecuencia, se plantean las siguientes cuatro hipótesis:

H6: Cuando compran un vino, los millennials limeños valoran más la producción orgánica y el precio que las otras generaciones.

$\mathrm{H}_{7}$ : Cuando compran un vino, los millennials limeños valoran más el diseño de la etiqueta y la botella que las otras generaciones.

H8: Cuando compran un vino, los millennials limeños valoran más el nivel de alcohol que las otras generaciones.

H9: Cuando compran un vino, los millennials limeños valoran menos la denominación de origen y el tiempo de envejecimiento que las otras generaciones.

\section{Importancia de los atributos a nivel de los estratos socioeconómicos}

Bernabéu et al. (2012) también analizó las preferencias del consumidor de vinos español por nivel de ingresos familiares, donde el segmento de bajos ingresos resultó estar más condicionado por el precio y las recomendaciones, mientras que los segmentos medios y altos fueron más condicionados por la región de origen y la variedad de uva. Además, los consumidores de altos ingresos valoraron más los vinos orgánicos, los cuales suelen tener un precio más alto que los vinos convencionales. Con el objetivo de analizar los atributos más importantes a nivel de los estratos socioeconómicos, se plantean las siguientes dos hipótesis:

H10: Cuando compran un vino, los consumidores limeños de bajos ingresos consideran que las recomendaciones son más importantes, mientras que el país de origen y la variedad de uva son menos importantes, en comparación a los consumidores limeños de medios y altos ingresos.

H11: Cuando compran un vino, los consumidores limeños de altos ingresos consideran que la producción orgánica es más importante, mientras que el precio es menos importante, en comparación a los consumidores limeños de bajos y medios ingresos.

\section{Importancia de los atributos a nivel de género}

Atkin et al. (2007) examinó diferencias de género en los criterios de elección relativos a la compra de vino. Las mujeres prefieren buscar información en el punto de venta, por medio de las etiquetas traseras y recomendaciones. Adicionalmente, se ha 
observado que las mujeres muestran una mayor tendencia a comprar en base a criterios secundarios, tales como las medallas ganadas, el precio, y el diseño del empaque (Thomas \& Pickering, 2003). Además, Bernabéu et al. (2012) observó una mayor preocupación en las mujeres por la producción orgánica del vino. Por lo tanto, se proponen las últimas dos hipótesis:

H12: Cuando compran vino, las mujeres limeñas valoran más las recomendaciones que los hombres limeños.

H13: Cuando compran vino, las mujeres limeñas valoran más el diseño del empaque y la producción orgánica que los hombres limeños.

\section{Técnicas usadas para medir la importancia de los atributos}

Lockshin y Hall (2003) revisaron más de 75 estudios relacionados al comportamiento del consumidor de vino, y analizaron las técnicas más frecuentes utilizadas en la industria para medir sus preferencias. Éstas técnicas son:

a. El panel de consumidores es una de las técnicas más frecuentes y de mayor alcance en la industria del vino, sin embargo, tiene algunas limitaciones en la medición de preferencias (Cohen, 2009). Si bien evalúa el vino que el consumidor ha comprado, no es necesariamente el vino que el consumidor hubiera deseado comprar. Esto podría enmascarar las preferencias reales. Por ejemplo, si un vino de Chardonnay está disponible para la venta cinco veces más que un vino de Sauvignon Blanc, esto no indica realmente que los consumidores prefieren el vino de Chardonnay (Goodman et al., 2005).

b. El análisis conjunto permite explorar y cuantificar actitudes para predecir preferencias (Green \& Rao, 1971). En el análisis, los encuestados informan su preferencia global para un perfil de producto determinado, con un número limitado de atributos, y con múltiples niveles. Luego, se estima la importancia relativa de cada uno de los atributos. Este método presenta varias ventajas, como los tradeoffs que los consumidores realizan al evaluar las opciones. Sin embargo, la complejidad en el diseño es una de sus principales desventajas (Goodman et al., 2005), y con muchas opciones al frente, los encuestados tienden a establecer estrategias de simplificación (Sattler \& Hensel-Boner, 2000). Esta técnica es apropiada para evaluar las combinaciones de atributos y los niveles de mayor interés para los consumidores. Al respecto, Talledo y Sánchez (2007) demuestran que el MaxDif y el análisis conjunto no miden lo mismo.

c. El modelo de elección discreta mide la utilidad de los atributos en diversas combinaciones llamadas conceptos de producto (Louviere \& Woodworth, 1983; Louviere, Hensher, \& Swait, 2000). Se calculan a partir de las elecciones realizadas, por lo tanto, es un método indirecto para medir preferencias (Louviere \& Islam, 2004). Este método brinda información útil con muestras pequeñas (Goodman et al., 2005), pero su problema es la complejidad del diseño, la dificultad de interpretar los datos, y de comparar utilidades de estudios distintos (Louviere et al., 2000).

d. Los investigadores también utilizan diferentes tipos de escala para medir preferencias. Algunas de ellas presentan limitaciones. Por ejemplo, en la escala de ranking, los encuestados ordenan los atributos en términos de importancia (Cohen, 2009). La tarea es relativamente fácil si el número de atributos es pequeño. A medida que el número de atributos aumenta la tarea se vuelve excesivamente agotadora (Weller \& Romney, 1988). La tarea de clasificación podría simplificarse mediante el uso de comparaciones pareadas, que es el método más confiable para la clasificación de ranking (Cohen \& Orme, 2004). La desventaja es que el número de pares se eleva rápidamente a medida que el número de atributos aumenta. Por ejemplo, para diez ítems se necesitan 45 pares de comparación, con 13 ítems se necesitan 78 pares (Cohen, 2009). Una forma típica de escala de rating es la tipo Likert. Un problema que suele presentarse en esta escala es que no todos los encuestados interpretan los descriptores de la misma manera, lo cual influye en la distancia percibida entre ellos (Crask \& Fox, 1987). Además, las personas pueden limitar sus respuestas a determinadas partes de la escala de rating (Bachman \& O'Malley, 1984). Esta fuente de error proviene del hecho de utilizar la escala en forma diferente (Goodman, Lockshin, \& Cohen, 2008), y los resultados pueden estar sujetos a sesgos que entregan puntuaciones difíciles de interpretar 
(Cohen, 2003; Cohen \& Neira, 2003; Cohen \& Orme, 2004; Finn \& Louviere, 1992). Sus diferencias pueden tener origen en el uso de la escala más que en las preferencias reales de los consumidores (Cohen, 2003). Otro problema es que usualmente cada atributo se mide con una escala de un solo ítem, por lo tanto, la confiabilidad y la validez son desconocidas (Goodman et al., 2005). La importancia de los atributos no se mide con relación a otros atributos, incluso si deben competir por los mismos recursos (Goodman et al., 2005). Tales respuestas no entregan la discriminación adecuada para identificar las prioridades reales de los consumidores (Finn \& Louviere, 1992), dado que no existe la posibilidad de realizar trade-offs entre los atributos.

\section{Método de máximas diferencias (MaxDif)}

El método de máximas diferencias (MaxDif), también conocido como el enfoque BW, es una extensión de las comparaciones pareadas que modela el proceso cognitivo por el cual los encuestados identifican sus preferencias sobre un conjunto de atributos organizados en subgrupos de tres o más elementos (Goodman et al., 2005). Fue desarrollado por Louviere y Woodworth (1990), y publicado por primera vez por Finn y Louviere (1992). Este enfoque asume la existencia de una dimensión subjetiva subyacente, como el grado de importancia o de preferencia, y el investigador desea medir la ubicación de un conjunto de los atributos a lo largo de aquella dimensión (Auger, Devinney, \& Louviere, 2004). La escala produce un ranking de los atributos para cada encuestado, y una escala en función a la muestra o la respuesta agregada de un segmento (Louviere \& Woodworth, 1990).

Siempre y cuando su diseño experimental esté balanceado, sumando el número total de veces que un atributo es elegido como mejor, y restando el número total de veces que se elige como peor, la técnica proporciona una escala tan precisa como un logit multinomial (MNL) para modelar los mismos datos (Auger et al., 2004). Entre sus principales ventajas están la alta diferenciación en el grado de importancia que los encuestados otorgan a los atributos, la prevención de sesgos de uso en evaluaciones diferentes (Casini et al., 2009), es especialmente apropiado para realizar comparaciones entre segmentos de consumidores (Cohen, 2009; Cohen \& Neira, 2003; Flynn et al., 2008; Goodman et al., 2005; Goodman, Lockshin, \& Cohen, 2008; Lee, Soutar, \& Louviere, 2008), y es simple de usar y entender (Goodman et al., 2005), y por lo tanto, este método es apropiado para su aplicación en esta investigación.

\section{DISEÑO DE LA INVESTIGACIÓN}

\section{Medición}

El cuestionario final presentó la siguiente estructura: (1) consumo y conocimiento de vinos, (2) escala MaxDif, (3) declaraciones de hábitos de consumo, (4) disposición a pagar por un vino orgánico, (5) características de los consumidores (edad, género, ingreso familiar mensual). Se realizó un estudio cualitativo exploratorio por medio de entrevistas a dos expertos en Lima, Perú para sondear la relevancia de los atributos propuestos en la revisión de la literatura. Los resultados reafirmaron la necesidad de utilizar 13 atributos. Si bien el MaxDif es un método reciente, ha sido ampliamente utilizado en investigaciones de diversos campos, por ejemplo en salud (Lancsar, Louviere, Donaldson, Currie, \& Burgess, 2013; Leung, 2013; Flynn, 2010; Flynn et al., 2007, 2008; Ryan, Amaya-Amaya, \& Gerard, 2008) y marketing de vinos (Bernabéu et al., 2012; Casini et al., 2009; Cohen, 2009; De-Magistris et al., 2014; Goodman et al., 2005; Lockshin, Cohen, \& Goodman, 2008), entre otros. Esto nos permite confiar en el MaxDif, que junto a una minuciosa selección de los 13 atributos, asegura que los resultados sean relevantes y representativos de las preferencias.

Quienes utilizan el método de máximas diferencias emplean el diseño de bloques incompletos balanceados (BIB) para organizar los atributos en subconjuntos o bloques de elección (p.ej., Casini et al., 2009; Cohen, 2009; De-Magistris et al., 2014). Así un gran número de atributos (p.ej., 13) pueden estudiarse con un número relativamente pequeño de bloques. Por lo tanto, en este trabajo se aplica un diseño BIB para colocar los 13 atributos en los bloques. El diseño BIB se denota como (b, $r, k, t)$, donde $b$ es el número de bloques, $r$ es el número de repeticiones por atributo en los bloques, k es el número de atributos en cada bloque, y t es el número de veces que dos atributos se topan en un bloque. Cohen (2009) considera que 13 bloques es un número que los encuestados pueden manejar, que 4 debe ser el número de repeticiones por atributo en los bloques para no generar un cuestionario muy extenso, que 4 debe ser el número de atributos que se deben incluir en cada bloque (un mayor número puede ser difícil de responder para los encuestados), y que solo una vez dos atributos se pueden topar en un bloque. Se realiza también una aleatorización del orden de los atributos, evitando sesgos de orden en los bloques. Por lo tanto, siguiendo a Cohen (2009) el diseño utilizado en este estudio fue un BIB (13, 4, 4, 1). Este diseño $\operatorname{BIB}(13,4,4,1)$ fue generado con el software $R$ obteniéndose los 13 bloques presentados en la Tabla 1. El Cuadro 1 presenta el bloque 1 del diseño BIB $(13,4,4,1)$ utilizado en esta investigación. 
Tabla 1. Bloques generados en el diseño $\mathrm{BIB}(\mathbf{1 3}, \mathbf{4}, \mathbf{4}, \mathbf{1})$

\begin{tabular}{|c|c|c|c|c|}
\hline Bloque & Atributo 1 & Atributo 2 & Atributo 3 & Atributo 4 \\
\hline 1 & 1 & 2 & 4 & 10 \\
\hline 2 & 2 & 3 & 5 & 11 \\
\hline 3 & 3 & 4 & 6 & 12 \\
\hline 4 & 4 & 5 & 7 & 13 \\
\hline 5 & 5 & 6 & 8 & 1 \\
\hline 6 & 6 & 7 & 9 & 2 \\
\hline 7 & 7 & 8 & 10 & 3 \\
\hline 8 & 8 & 9 & 11 & 4 \\
\hline 9 & 9 & 10 & 12 & 5 \\
\hline 10 & 10 & 11 & 13 & 6 \\
\hline 11 & 11 & 12 & 1 & 7 \\
\hline 12 & 12 & 13 & 2 & 8 \\
\hline 13 & 13 & 1 & 3 & 9 \\
\hline
\end{tabular}

\section{Cuadro 1. Bloque 1 del diseño BIB $(13,4,4,1)$}

Por favor marque el atributo más importante y el menos importante que tomaría en cuenta cuando compra/elige un vino.

Para cada bloque, marque solo una opción en la columna "Más importante" y sola una opción en la columna "Menos importante".

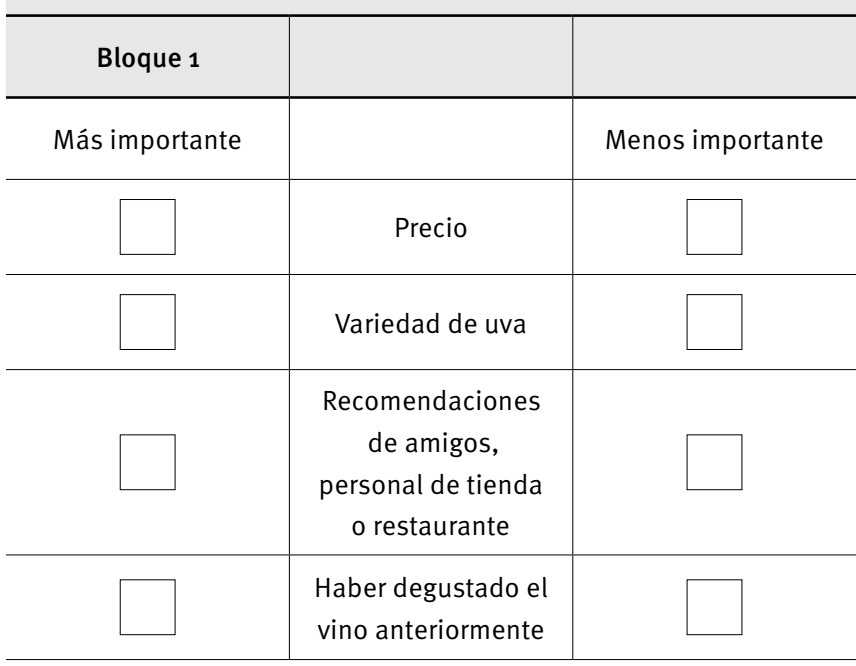

Para medir la confiabilidad del MaxDif, se aplicó el cuestionario a 25 encuestados. Después de una semana, se aplicó nuevamente el cuestionario a las mismas personas. El análisis se realizó con los puntajes BW obtenidos para cada uno de los atributos. Los resultados obtenidos muestran un alto grado de confiabilidad para la escala, con un coeficiente de correlación de Pearson que alcanza el o,97.

\section{Muestra}

Los datos fueron recolectados enviando un cuestionario online, usando Qualtrics, a una base de consumidores de vino de la ciudad de Lima, Perú. Se recolectaron 260 encuestas finalizadas durante los meses de Diciembre de 2014 y Enero de 2015. La Tabla 2 muestra la descripción de la muestra. El 49\% de la muestra corresponde a mujeres, cifra muy cercana a la observada en la población de Lima (51\%) y a nivel de Perú (50\%). Adicionalmente, el $37 \%$ de la muestra tiene entre 18 y 34 años, cifra muy cercana a la observada en Lima (39\%) y en Perú (40\%) (INEI, 2015). El $31 \%$ de la muestra es posible clasificarla como consumidores de bajos ingresos, cifra cercana a la observada en Lima (33\%), pero más alejada a la observada a nivel de Perú (45\%) (Perú21, 2013). Por lo tanto, es posible concluir que la muestra es bastante representativa de la ciudad de Lima. Asimismo, la muestra es bastante heterogénea en las tres variables de segmentación lo que permitirá analizar correctamente las hipótesis propuestas en esta investigación.

\section{Tabla 2. Descripción de la muestra}

\begin{tabular}{|c|c|c|}
\hline Variable & \multirow{2}{*}{$\mathbf{N}$} & \multirow{2}{*}{$\%$} \\
\hline Género & & \\
\hline Masculino & 132 & 51 \\
\hline Femenino & 128 & 49 \\
\hline Edad & $\mathbf{N}$ & $\%$ \\
\hline $18-34$ & 95 & 37 \\
\hline $35-49$ & 88 & 34 \\
\hline $50-64$ & 77 & 30 \\
\hline Ingreso familiar mensual en S/. & $\mathbf{N}$ & $\%$ \\
\hline$<5.000$ & 81 & 31 \\
\hline $5.000>10.000$ & 89 & 34 \\
\hline$>10.000$ & 90 & 35 \\
\hline Total & 260 & 100 \\
\hline
\end{tabular}

\section{Análisis de los datos}

Para el análisis de los datos se utilizó la puntuación BW (BWscore), la cual permitirá obtener un ranking de los atributos en términos 
de importancia (Cohen, 2009; Cohen \& Neira, 2003; Goodman et al., 2005). La puntuación BW se obtiene restando el número de veces que un atributo es elegido como el menos importante (W, Worst) del número de veces que el atributo es elegido como el más importante (B, Best). Como cada atributo es incluido 4 veces en los bloques en el diseño BIB $(13,4,4,1)$ (ver Tabla 1), la puntuación BW para cada atributo puede ir desde un mínimo de -4 (4 veces es elegido el peor atributo) a un máximo de +4 (4 veces es elegido el mejor atributo). La puntuación BW promedio para cada atributo se obtiene promediando los puntajes BW para cada atributo entre los encuestados. Esta puntuación también se puede estandarizar dividiendo por 4. Otra forma de analizar la importancia de los atributos es por medio de la raíz cuadrada de la división (B/W). Este coeficiente mide la probabilidad de elección respecto al atributo más importante con 100\% (Auger, Devinney, \& Louviere, 2007; Flynn et al., 2007; Lee et al., 2008; Marley \& Louviere, 2005). Además, si el atributo más importante se convierte en 100, se pueden estandarizar los datos (Jaeger, Danaher, \& Brodie, 2009; Jaeger, Jørgensen, Aaslyng, \& Bredie, 2008). El resultado se interpreta como $X \%$ de la probabilidad de ser elegido como el más importante.

Para responder si un atributo es significativamente diferente a otro puede aplicarse un ANOVA de un factor. Así, pueden observarse grupos estadísticamente diferentes que surgen dentro de los datos (Cohen, 2009). En esta investigación, se efectuarán ANOVAs de un factor para analizar las diferencias de medias entre atributos y segmentos. Para la segmentación, la variable ingresos se dividió en tres rangos: bajos ( $45,000 \mathrm{~s} /$.$) , medios (5,000-10,000$ s/.), y altos (>10,000 s/.); la variable edad se dividió en 18-34 años (millennials), 35-49 años (generación X), y 50-64 años (baby boomers); y el género en masculino y femenino.

\section{RESULTADOS}

\section{A nivel de la ciudad de Lima, Perú}

A nivel de la ciudad de Lima, Perú (ver Tabla 3) destacan los atributos relacionados al haber degustado el vino previamente con 1,57 , el maridaje con 1,30, y las recomendaciones de amigos y personal especializado $(1,07)$. El consumidor limeño valora mucho el haber degustado el vino previamente y las recomendaciones cuando elige un vino. De ahí el rol protagónico del personal de contacto (sommelier y vendedores) para efectuar degustaciones y recomendaciones relevantes para el consumidor. Estos resultados soportan las Hipótesis 1 y 2 . Por otro lado, el diseño de la botella y etiqueta $(-1,94)$, y el nivel de alcohol $(-1,35)$ son los atributos menos importantes para la compra de vino.
Tabla 3. Importancia de los atributos a nivel de la ciudad de Lima, Perú

\begin{tabular}{l|c}
\hline Atributo & BWscore \\
\hline Haber degustado el vino anteriormente & 1,57 \\
\hline Va con la comida/maridaje & 1,30 \\
\hline $\begin{array}{l}\text { Recomendaciones de amigos, personal de tienda o } \\
\text { restaurante }\end{array}$ & 1,07 \\
\hline Tiempo de envejecimiento & 0,68 \\
\hline Variedad de uva & 0,48 \\
\hline Producción orgánica & 0,20 \\
\hline La marca & 0,17 \\
\hline Denominación de origen & $-0,40$ \\
\hline País de origen & $-0,53$ \\
\hline Oferta/Promoción/Descuento de la tienda o restaurante & $-0,62$ \\
\hline Precio & $-0,61$ \\
\hline Nivel de alcohol menor a 13\% & $-1,35$ \\
\hline Diseño de la botella y etiqueta & $-1,94$ \\
\hline
\end{tabular}

Para el consumidor limeño la variedad de uva $(0,48)$ está entre los cinco atributos más importantes. Se debe señalar que la variedad de uva puede tratarse de una variedad tipo Cabernet Sauvignon, o simplemente blanca o tinta, lo cual dependerá del grado de conocimiento del consumidor que realiza la compra. Por lo tanto, los resultados soportan la Hipótesis 3.

En contraste, es posible observar que el país de origen $(-0,53)$ no es un atributo importante para los consumidores limeños al momento de comprar vino. Por lo tanto, la Hipótesis 4 no es soportada.

La oferta/promoción/descuento $(-0,62)$ no es tan determinante como otros atributos. Este resultado quizás es consecuencia de la gran cantidad de vinos en promoción en el canal off-trade, que concentra el $80 \%$ del mercado de vino en Perú. Por lo tanto, la Hipótesis 5 no es soportada.

En resumen, los resultados permiten concluir que las preferencias de los consumidores limeños tienden a ser más similares a las encontradas en los consumidores de países emergentes. Sin embargo, destaca el maridaje como un atributo muy valorado tanto por los consumidores limeños como por los consumidores de países desarrollados. Si bien la gastronomía peruana ha tenido un desarrollo importante en los últimos años, el hábito de combinarla con los vinos es aún incipiente. Los resultados muestran una gran oportunidad de crecimiento en este aspecto. 


\section{A nivel de generaciones etarias}

A nivel de generaciones etarias (ver Tabla 4), los jóvenes buscan asegurar su elección apoyándose en personas con más experiencia por medio de recomendaciones $(0,66 ; p<, 05), y$ luego giran a la propia experiencia de haber degustado el vino previamente $(0,58)$. En contraste, los consumidores de mayor edad son más fieles a sus vinos favoritos, debido a que el atributo más importante en ese segmento es haber degustado el vino previamente $(0,51)$. Esto demuestra que los jóvenes tienden a ser más aventureros en el vino, y por ende, con una mayor predisposición a experimentar dentro de un rango de marcas conocidas o recomendadas $(0,15)$.

\section{Tabla 4. Importancia de los atributos a nivel de generaciones etarias}

\begin{tabular}{|c|c|c|c|}
\hline & $\begin{array}{c}\text { Millennials } \\
(18-34)\end{array}$ & $\begin{array}{c}\text { Generación } \\
X(35-49)\end{array}$ & $\begin{array}{c}\text { Baby } \\
\text { Boomers } \\
(50-64)\end{array}$ \\
\hline Atributo & BWscore & BWscore & BWscore \\
\hline $\begin{array}{l}\text { Denominación de } \\
\text { origen* }\end{array}$ & $-0,29$ & $-0,09$ & $-0,02$ \\
\hline $\begin{array}{l}\text { Diseño de la botella } \\
\text { y etiqueta* }\end{array}$ & $-0,54$ & $-0,66$ & $-0,74$ \\
\hline $\begin{array}{l}\text { Haber degustado el } \\
\text { vino anteriormente }\end{array}$ & 0,58 & 0,47 & 0,51 \\
\hline La marca & 0,15 & $-0,02$ & 0,04 \\
\hline $\begin{array}{l}\text { Nivel de alcohol } \\
\text { menor a } 13 \%\end{array}$ & $-0,52$ & $-0,37$ & $-0,47$ \\
\hline $\begin{array}{l}\text { Oferta/Promoción/ } \\
\text { Descuento de la } \\
\text { tienda o restaurante }\end{array}$ & $-0,15$ & $-0,20$ & $-0,27$ \\
\hline País de origen & $-0,20$ & $-0,20$ & $-0,13$ \\
\hline Precio & $-0,23$ & $-0,24$ & $-0,13$ \\
\hline Producción orgánica* & $-0,09$ & 0,05 & 0,25 \\
\hline $\begin{array}{l}\text { Recomendaciones de } \\
\text { amigos, personal de } \\
\text { tienda o restaurante* }\end{array}$ & 0,66 & 0,28 & 0,12 \\
\hline $\begin{array}{l}\text { Tiempo de } \\
\text { envejecimiento }\end{array}$ & 0,14 & 0,28 & 0,25 \\
\hline $\begin{array}{l}\text { Va con la comida/ } \\
\text { maridaje* }\end{array}$ & 0,45 & 0,52 & 0,33 \\
\hline Variedad de uva* & 0,04 & 0,18 & 0,26 \\
\hline
\end{tabular}

*Indica diferencias entre grupos estadísticamente significativas al 5\%.
La Hipótesis 6 no es soportada. La producción orgánica $(-0,09)$ y el precio $(-0,23)$ no son más importantes para los jóvenes. La producción orgánica se vuelve más determinante conforme se incrementa la edad, y con ello la preocupación por la salud. Por otro lado, el precio es más importante para los baby boomers $(-0,13)$. Eso no indica que compren vinos más económicos, sino que evalúan el precio con mayor detalle para verificar que el vino esté conforme a la situación de consumo.

En general, el diseño de la botella y etiqueta es el atributo menos importante en la compra, ligeramente más influyente para los millennials $(-0,54)$. Por lo tanto, la Hipótesis 7 no es soportada.

La Hipótesis 8 no es soportada. El nivel de alcohol es poco relevante para la generación millennials $(-0,52)$. Sin embargo, esto puede cambiar en el futuro. En países como Estados Unidos e Inglaterra, vinos de bajo grado alcohólico están teniendo mucho éxito, sobretodo en el público joven femenino. A esto se suma una mayor conciencia por la salud y regulaciones de tránsito para prevenir accidentes.

La Hipótesis 9 es soportada. En forma similar a García et al. (2013) y De-Magistris et al. (2014), los jóvenes están menos interesados en la denominación de origen $(-0,29 ; p<0,05)$ y el tiempo de envejecimiento $(0,14)$ que las otras generaciones. Esto sugiere la no necesidad de mayor comunicación e información para incrementar el conocimiento de los jóvenes de estos dos atributos, sino por el contrario, la simplificación en la comunicación hacia los atributos más importantes.

\section{A nivel de estratos socioeconómicos}

La Tabla 5 muestra un análisis de los atributos más importantes a nivel de estratos socioeconómicos. Las personas de mayores ingresos valoran más las recomendaciones (0,41 y 0,42; $p<0,05)$, y el país de origen $(-0,04 ; p<0,05)$, por un tema de accesibilidad a canales de comercialización on-trade y off-trade con diversidad de estos atributos. Estos resultados no soportan la Hipótesis 10.

Las personas de mayores ingresos valoran menos la producción orgánica $(-0,02)$, y valoran más otros atributos como el precio $(-0,07 ; \mathrm{p}<0,05)$ y las ofertas, promociones y descuentos $(-0,04)$. Al tener mayor accesibilidad a productos con gran diversidad de precios y promociones, los consumidores se ven atraídos a realizar comparaciones entre ambos atributos con el objetivo de encontrar buenas oportunidades en la compra. Estos resultados no soportan la Hipótesis 11.

\section{A nivel de género}

La Tabla 6 muestra un análisis de los atributos más importantes para cada género. La Hipótesis 12 es soportada parcialmente. 
Efectivamente, las mujeres valoran más las recomendaciones $(0,58)$ que los hombres $(0,48)$, sin embargo, esta diferencia no es estadísticamente significativa. La Hipótesis 13 es soportada al observarse que la producción orgánica $(0,19 ; p<0,05)$ y el nivel de alcohol $(-0,47 ; p<0,05)$ son atributos más importantes para las mujeres. Esto posiblemente porque las mujeres buscan un estilo de vida más saludable que los hombres.

Tabla 5. Importancia de los atributos a nivel de estratos socioeconómicos

\begin{tabular}{|c|c|c|c|}
\hline & $\begin{array}{c}\text { Estrato } \\
\text { Bajo }\end{array}$ & $\begin{array}{l}\text { Estrato } \\
\text { Medio }\end{array}$ & $\begin{array}{c}\text { Estrato } \\
\text { Alto }\end{array}$ \\
\hline Atributo & BWscore & BWscore & BWscore \\
\hline Denominación de origen & $-0,13$ & $-0,19$ & $-0,08$ \\
\hline $\begin{array}{l}\text { Diseño de la botella y } \\
\text { etiqueta }\end{array}$ & $-0,58$ & $-0,70$ & $-0,66$ \\
\hline $\begin{array}{l}\text { Haber degustado el vino } \\
\text { anteriormente }\end{array}$ & 0,50 & 0,53 & 0,53 \\
\hline La marca & $-0,03$ & 0,12 & 0,08 \\
\hline $\begin{array}{l}\text { Nivel de alcohol menor } \\
\text { a } 13 \% \star\end{array}$ & $-0,17$ & $-0,56$ & $-0,62$ \\
\hline $\begin{array}{l}\text { Oferta/Promoción/ } \\
\text { Descuento de la tienda o } \\
\text { restaurante }\end{array}$ & $-0,30$ & $-0,18$ & $-0,14$ \\
\hline País de origen* & $-0,17$ & $-0,32$ & $-0,04$ \\
\hline Precio* & $-0,35$ & $-0,19$ & $-0,07$ \\
\hline Producción orgánica & 0,12 & 0,10 & $-0,02$ \\
\hline $\begin{array}{l}\text { Recomendaciones de } \\
\text { amigos, personal de } \\
\text { tienda o restaurante* }\end{array}$ & 0,24 & 0,42 & 0,41 \\
\hline $\begin{array}{l}\text { Tiempo de } \\
\text { envejecimiento* }\end{array}$ & 0,21 & 0,36 & 0,11 \\
\hline $\begin{array}{l}\text { Va con la comida/ } \\
\text { maridaje }\end{array}$ & 0,49 & 0,49 & 0,32 \\
\hline Variedad de uva & 0,17 & 0,12 & 0,19 \\
\hline
\end{tabular}

*Indica diferencias entre grupos estadísticamente significativas al 5\%.
Tabla 6. Importancia de los atributos a nivel de género

\begin{tabular}{|c|c|c|}
\hline & Hombres & Mujeres \\
\hline Atributo & BWscore & BWscore \\
\hline Denominación de origen* & $-0,01$ & $-0,39$ \\
\hline Diseño de la botella y etiqueta & $-1,00$ & $-0,94$ \\
\hline Haber degustado el vino anteriormente & 0,71 & 0,86 \\
\hline La marca & 0,11 & 0,06 \\
\hline Nivel de alcohol menor a 13\%* & $-0,89$ & $-0,47$ \\
\hline $\begin{array}{l}\text { Oferta/Promoción/Descuento de la } \\
\text { tienda o restaurante }\end{array}$ & $-0,36$ & $-0,26$ \\
\hline País de origen* & $-0,03$ & $-0,50$ \\
\hline Precio & $-0,25$ & $-0,36$ \\
\hline Producción orgánica* & 0,01 & 0,19 \\
\hline $\begin{array}{l}\text { Recomendaciones de amigos, personal } \\
\text { de tienda o restaurante }\end{array}$ & 0,48 & 0,58 \\
\hline Tiempo de envejecimiento & 0,30 & 0,38 \\
\hline Va con la comida/maridaje & 0,67 & 0,64 \\
\hline Variedad de uva & 0,27 & 0,20 \\
\hline
\end{tabular}

\section{CONCLUSIONES E IMPLICANCIAS}

\section{Implicancias para administradores}

Este estudio ha ilustrado que el método de máximas diferencias es simple de diseñar, implementar e interpretar para analizar las preferencias del consumidor. Por medio del análisis de las puntuaciones BW, el investigador puede medir las preferencias del consumidor, evitando sesgos en la escala, y logrando realizar comparaciones entre segmentos de consumidores. A nivel de la ciudad de Lima, los resultados de esta investigación sugieren las siguientes recomendaciones:

- Incrementar las oportunidades de degustación del vino en tiendas, en restaurantes y eventos relacionados al vino y la gastronomía, y también no relacionados (deportivos, sociales, artísticos, etc.). La degustación incrementa la probabilidad de compra. 
- Combinar la gastronomía local con el vino. Esto implica cierto nivel de adaptación en el producto (etiqueta trasera), y las comunicaciones. También se recomienda formar alianzas con el canal on-trade.

- El personal de contacto debe estar capacitado para dar recomendaciones relevantes para el consumidor, entorno a señales de alta influencia en las decisiones de compra. Por ejemplo, qué variedades de uva combinan mejor con los platos locales.

- Poner énfasis en la marca, por medio de la comunicación de elementos atractivos y la participación en eventos. Las visitas del enólogo o embajadores de marca contribuyen a estrechar vínculos con el consumidor local que aprecia el vino.

Además de lo anterior, para enfocar el marketing hacia los millennials, se recomienda:

- Simplificar la información de las etiquetas frontales, respecto a la denominación de origen y tiempo de envejecimiento. Este grupo de consumidores no está interesado en aquellos atributos, y pueden generar confusión en el proceso de compra.

- La marca puede ser muy relevante en este segmento. Por ello, se recomienda poner énfasis en comunicar temáticas menos tradicionales entorno al vino, y resaltar la faceta social y de entretenimiento. Esto debe venir de la mano con el uso de medios digitales.

- Es el segmento ideal para introducir innovaciones al mercado, desde niveles de azúcar y alcohol, hasta empaques (latas, pouch, tetra brik, single serve, bagin-box, otros).

A nivel de ingresos, los resultados sugieren las siguientes implicancias:

- Enfocar la comunicación y las recomendaciones en variedades de uva internacionales y el país de origen para el segmento de altos ingresos. Estos consumidores pueden recibir bien vinos de variedades poco usuales en el mercado, como el Riesling y Pinot Noir, las cuales entregan un alto grado de sofisticación al consumidor. Este segmento aprecia las promociones y descuentos que ofrecen los diversos canales, entre ellos clubs de puntos, entre otros, y poder visitar las bodegas como turista.

- Los segmentos de bajos ingresos prefieren las variedades locales. Esto no impide que bodegas extranjeras puedan ofrecer alternativas similares que se adapten bien al gusto local y puedan venderse a precios competitivos.

- Controlar el uso de malas prácticas comerciales en el canal off-trade, dado que algunos minoristas se pueden aprovechar del bajo conocimiento del consumidor para ofrecer vinos en promoción que han sobrepasado el tiempo ideal de envejecimiento. La marca y la imagen del país de origen pueden verse afectadas cuando el consumidor compra y consume estos productos que no están en su estado óptimo.

Para enfocar el marketing hacia las mujeres, los resultados sugieren las siguientes recomendaciones:

- Simplificar atributos como la denominación de origen, en favor de resaltar otros como el diseño de las etiquetas, las certificaciones orgánicas y el nivel de alcohol.

- Vinos de producción orgánica y/o menores niveles de alcohol pueden ser particularmente atractivos para este segmento. Actualmente, el mercado peruano tiene pocas opciones enfocadas en este grupo con alto potencial de crecimiento.

- Las promociones y descuentos pueden tener buenos resultados en las ventas, siempre y cuando los productos puedan satisfacer primero los atributos más importantes para este segmento.

\section{Limitaciones y futuras líneas de investigación}

Pese a que la muestra obtenida permitió alcanzar los objetivos de la investigación, una limitación del estudio fue el pequeño tamaño de la muestra y el enfoque en la ciudad de Lima. Futuras investigaciones pueden evaluar preferencias en distintas zonas o regiones del país para crear una base comparativa nacional. Este trabajo también anima a realizar este análisis en otros mercados latinoamericanos con el objetivo de analizar diferencias y similitudes con los resultados encontrados en esta investigación. 
A pesar de seleccionar cuidadosamente los atributos por medio de la literatura internacional y la opinión de expertos locales, es posible que existan otros atributos importantes, y si uno incluye o borra un atributo, los resultados pueden variar. Otra línea de investigación interesante sería investigar a mayor profundidad algunos atributos señalados en este estudio; por ejemplo, las preferencias sobre variedades de uva o marcas, y analizar con mayor profundidad ciertos segmentos con un alto potencial de crecimiento como son los consumidores millennials y las mujeres. Finalmente, se puede replicar el estudio para observar la evolución de las preferencias encontradas o realizar análisis con otros diseños BIB o técnicas usadas para medir la importancia de los atributos (p.ej., análisis conjunto).

\section{AGRADECIMIENTOS}

El primer autor agradece el apoyo para el proyecto FONDECYT N 11130614. Los autores agradecen a los editores y revisores por la orientación y retroalimentación.

\section{REFERENCIAS}

Arellano Marketing (2014). El 38\% de los peruanos prefieren las ofertas al momento de hacer compras (en linea). Recuperado de http://www. arellanomarketing.com/inicio/el-38-de-los-peruanosprefieren-lasofertas-al-momento-de-hacer-sus-compras/

Atkin, T., Nowak, L., \& Garcia, R. (2007). Women wine consumers: Information search and retailing implications. International Journal of Wine Business Research, 19(4), 327-339. doi:10.1108/17511060710837454

Auger, P., Devinney, T. M., \& Louviere, J. J. (2004). Consumer's social beliefs, an international investigation using best worst scaling methodology [Working Paper]. University of Melbourne, Melbourne Business School, Melbourne.

Auger, P., Devinney, T. M., \& Louviere, J. J. (2007). Using best-worst scaling methodology to investigate consumer ethical beliefs across countries. Journal of Business Ethics, 70(3), 299-326. doi:10.1007/ S10551-006-9112-7

Bachman, J. G., \& O’Malley, P. M. (1984). Yea-saying, nay-saying, and going to extremes: Black-White differences in response styles. The Public Opinion Quarterly, 48(2), 491-509.

Balestrini, P., \& Gamble, P. (2006). Country-of-origin effects on Chinese wine consumers. British Food Journal, 108(5), 396-412. doi:10.1108/00070700610661367

Batt, P. J., \& Dean, A. (2000). Factors influencing the consumer's decision. Australia and New Zealand Wine Industry Journal Marketing Supplement, 15(4), 34-41.

Bernabéu, R., Díaz, M., Olivas, R., \& Olmeda, M. (2012). Consumer preferences for wine applying best-worst scaling: A Spanish case study. British Food Journal, 114(9), 1228-1250. doi:10.1108/00070701211258790
Bruwer, J., Li, B., \& Reid, M. (2002). Segmentation of the Australian wine market using a wine-related lifestyle approach. Journal of Wine Research, 13(3), 217-242. doi:10.1080/0957126022000046510

Casini, L., Corsi, A. M., \& Goodman, S. (2009). Consumer preferences of wine in Italy applying best-worst scaling. International Journal of Wine Business Research, 21(1), 64-78. doi:10.1108/17511060910948044

Cohen, E. (2009). Applying best-worst scaling to wine marketing. International Journal of Wine Business Research, 21(1), 8-23. doi:10.1108/17511060910948008

Cohen, S. (2003). Maximum difference scaling: Improved measures of importance and preference for segmentation. Sawtooth Software Conference Proceedings, Sawtooth Software, Inc., 530 W. Fir St., Sequim, WA.

Cohen, S., \& Neira, L. (2003). Measuring preference for product benefits across countries: Overcoming scale usage bias with maximum difference scaling. Proceedings of Latin American Conference of the European Society for Opinion and Marketing Research, Punta del Este, Uruguay.

Cohen, S., \& Orme, B. (2004). What's your preferences? Market Research, 16(2), 32-37.

Crask, M. R., \& Fox, R. J. (1987). An exploration of the interval properties of three commonly used marketing research studies: A magnitude estimation approach. Journal of the Marketing Research Society, 29(3), 317-339.

De-Magistris, T., Gracia, A., \& Albisu, L. (2014). Wine consumers' preferences in Spain: An analysis using the best-worst scaling approach. Spanish Journal of Agricultural Research, 12(3), 529-541. doi:10.5424/sjar/2014123-4499

El Comercio (2014). ¿Cuántos litros de vino se consumen en el Perú cada año? Recuperado de http://elcomercio.pe/economia/peru/cuantoslitros-vino-se-consumen-peru-cada-ano-noticia-1743755

Farías, P. (2015). Determinants of the success of global and local brands in Latin America. RAE-Revista de Administração de Empresas, 55(5), 539-550. doi:10.1590/So034-759020150506

Felzensztein, C., Hibbert, S., \& Vong, G. (2004). Is the country of origin the fifth element in the marketing mix of imported wine? A critical review of the literature. Journal of Food Products Marketing, 10(4), 73-84. doi:10.1300/j038v10no4_05

Finn, A., \& Louviere, J. J. (1992). Determining the appropriate response to evidence of public concern: The case of food safety. Journal of Public Policy \& Marketing, 11(2), 12-15.

Flynn, T. (2010). Valuing citizen and patient preference in health: Recent developments in three types of best-worst scaling. Expert Review of Pharmacoeconomics \& Outcomes Research, 10(3), 259-267. doi:10.1586/erp.10.29

Flynn, T. N., Louviere, J., Peters, T. J., \& Coast, J. (2007). Best worst scaling: What it can do for health care research and how to do it. Journal of Health Economics, 26(1), 171-189. doi:10.1016/j. jhealec0.2006.04.002

Flynn, T. N., Louviere, J. J., Peters, T. J., \& Coast, J. (2008). Estimating preferences for a dermatology consultation using best-worst scaling: Comparison of various methods of analysis. BMC Medical Research Methodology, 8(76), 76, 1-12. doi:10.1186/1471-2288-8-76

García, T., Barrena, R., \& Grande, I.. (2013). The wine consumption preferences of young people: A Spanish case study. International Journal of Wine Business Research, 25(2), 94-107. doi:10.1108/ ijwbr-2012-0007 
Gestión (2013). El 85\% de vinos que se venden en Perú están por debajo de S/. 100. Recuperado de http://gestion.pe/empresas/85-vinosque-se-venden-peru-estan-debajo-s-100-2070159

Goodman, S. (2009). An international comparison of retail consumer wine choice. International Journal of Wine Business Research, 21(1), 41-49. doi:10.1108/17511060910948026

Goodman, S., Lockshin, L., \& Cohen, E. (2005, 8-9 July). Bestworst scaling: A simple method for determining drink and wine style preferences. Proceedings of International Wine Marketing Symposium, Sonoma, CA, 2.

Goodman, S., Lockshin, L., \& Cohen, E. (2008). Examining market segments and influencers of choice for wine using the best-worst choice method. Market Management: Marketing and Communication, 8(1), 94-112.

Green, P. E., \& Rao, V. R. (1971). Conjoint measurement for quantifying judgmental data. Journal of Marketing Research, 8(3), 355-363. doi:10.2307/3149575

Guzmán P. (2014). Concha y Toro venderá 200,000 cajas de vinos de alta gama en Perú (en línea). Recuperado de http://gestion.pe/ empresas/concha-y-toro-vendera-20000o-cajas-vinos-alta-gamaperu-2112624

Instituto Nacional de Estadística e Informática. (2014). Una mirada a Lima Metropolitana (en línea). Recuperado de http://www.inei.gob. pe/media/MenuRecursivo/publicaciones_digitales/Est/Lib1168/ libro.pdf

Instituto Nacional de Estadística e Informática (2015). Estimaciones y proyecciones de población departamental por años calendario y edades simples 1995-2025. Recuperado de http://proyectos.inei. gob.pe/web/biblioineipub/bancopub/Est/Lib1039/index.htm

Jaeger, S. R., Danaher, P. J., \& Brodie, R. J. (2009). Wine purchase decisions and consumption behaviours: Insights from a probability sample drawn in Auckland, New Zealand. Food Quality and Preference, 20(4), 312-319. doi:10.1016/j.foodqual.2009.02.003

Jaeger, S. R., Jørgensen, A. S., Aaslyng, M. D., \& Bredie, W. L. P. (2008). Best-worst scaling: An introduction and initial comparison with monadic rating for preference elicitation with food products. Food Quality and Preference, 19(6), 579-588. doi:10.1016/j. foodqual.2008.03.002

Lancsar, E., Louviere, J., Donaldson, C., Currie, G., \& Burgess, L. (2013). Best worst discrete choice experiments in health: Methods and an application. Social Science \& Medicine, 76, 74-82. doi:10.1016/j. socscimed.2012.10.007

Lee, J. A., Soutar, G., \& Louviere, J. J. (2008). The best-worst scaling approach: An alternative to Schwartz's values survey. Journal of Personality Assessment, 90(4), 335-347. doi:10.1080/00223890802107925

Leung, T. (2013). Validity and feasibility of best worst scaling using multiple treatment outcomes of Parkinson's disease. Master Graduation Thesis, School of Health Science, University of Twente.

Lockshin, L., \& Hall, J. (2003). Consumer purchasing behaviour for wine: What we know and where we are going. Paper presented at the International Wine Marketing Colloquium, University of South Australia, Adelaide.

Lockshin, L., Cohen, E., \& Goodman, S. (2008). Overcoming measurement errors: Segmenting wine consumers across 11 countries. Ehrenberg-Bass, Institute for Marketing Science, Wine Marketing Group, University of South Australia.

Louviere, J. J., Lings, I., Islam, T., Gudergan, S. \& Flynn, T.. (2013). An introduction to the application of (case 1) best-worst scaling in marketing research. International Journal of Research in Marketing, 30(3), 292-303. doi:10.1016/j.ijresmar.2012.10.002

Louviere, J. J., \& Islam, T. (2004, September). A comparison of importance weights/measures derived from choice-based conjoint, constant sum scales and best-worst scaling [Working Paper No. 04o03]. Ce,SoC.

Louviere J. J., \& Woodworth G. G. (1983). Design and analysis of simulated consumer choice allocation experiments: An approach based on aggregate data. Journal of Marketing Research, 20(4), 350367. doi:10.2307/3151440

Louviere, J. J., \& Woodworth, G. G. (1990). Best worst scaling: A model for largest difference judgments [Working Paper]. Faculty of Business, University of Alberta, Edmonton.

Louviere, J. J., Hensher, D. A., \& Swait, J. D. (2000). Stated choice methods: Analysis and application. Cambridge, UK: Cambridge University Press.

Manske, M., \& Cordua, G. (2005). Understanding the sommelier effect. International Journal of Contemporary Hospitality Management, 17(7), p569-576. doi:10.1108/09596110510620645

Manzur, E., Olavarrieta, S., Hidalgo, P., \& Farías, P. (2013). Store price promotion strategies: An empirical study from Chile. Academia Revista Latinoamericana de Administración, 26(3), 356-372. doi:10.1108/arla-07-2013-0093

Manzur, E., Olavarrieta, S., Hidalgo, P., Farías, P., \& Uribe, R. (2011). Store brand and national brand promotion attitudes antecedents. Journal of Business Research, 64(3), 286-291. doi:10.1016/j. jbusres.2009.11.014

Marley, A. A., \& Louviere, J. J. (2005). Some probabilistic models of best, worst and best-worst choices. Journal of Mathematical Psychology, 49(6), 464-480. doi:10.1016/j.jmp.2005.05.003

Olavarrieta, S., Hidalgo, P., Manzur, E., \& Farías, P. (2012). Determinants of in-store price knowledge for packaged products: An empirical study in a Chilean hypermarket. Journal of Business Research, 65(12), 1759-1766. doi:10.1016/j.jbusres.2011.10.035

Paulas, R. (2014). Marketing Zombie Zin and other wines for millennials (en línea). Recuperado de http://www.kcet.org/living/food/foodrant/marketing-zombie-zin-other-wines-for-millennials.html

Perú21 (2011). Consumo de vino se duplica y se refina. Recuperado de http://peru21.pe/noticia/1314604/consumo-vino-se-duplica-serefina

Perú21 (2013). El 55\% de los hogares urbanos pertenece a clase media y alta. Recuperado em Agosto 27, 2015, de http://peru21. pe/economia/55-hogares-urbanos-pertenece-clase-media-yalta-2146622

Ryan, M., Amaya-Amaya, M., \& Gerard, K. (2008). Using discrete choice experiments to value health and health care. Dordrecht: Elsevier.

Sattler, H., \& Hensel-Boner, S. (2000). A comparison of conjoint measurement with self-explicated approaches. In A. Gustafsson, A. Herrmann, \& F. Huber (Eds.). Conjoint measurement: Methods and applications. Berlin: Springer. 
Sociedad Nacional de Industrias. (2014). Industria de elaboración de vinos (en línea). Recuperado de http://www.sni.org.pe/?page_id $=872$

Talledo, H. \& Sánchez, J. (2007, 19, 20 y 21 Septiembre). Del análisis conjunto a las escalas de máximas diferencias: Un estudio comparativo entre Maxdiff y escalasmonádicas, desde la validez convergente y discriminante. In Escuela Superior de Gestión Comercial y Marketing, ESIC (Ed), XIX Encuentro de Profesores Universitarios de Marketing [recurso electrónico] organizado por la Escuela Superior de Gestión Comercial y Marketing, Vigo, España.
Thach, E., \& Olsen, J. E. (2006). Market segment analysis to target young adult wine drinkers. Agribusiness, 22(3), 307-322. doi:10.1002/ agr.20088

Thomas, A., \& Pickering, G. (2003). The importance of wine label information. International Journal of Wine Marketing, 15(2), 58-75. doi:10.1108/eboo8757

Weller, S. W., \& Romney, A. K. (1988). Systematic data collection. Newbury Park, CA: Sage. 